Toxicology

\title{
A perspective of mitochondrial dysfunction in rats treated with silver and titanium nanoparticles (AgNPs and TiNPs)
}

\author{
Lilian Cristina Pereira ${ }^{\mathrm{a}, \mathrm{b}, *}$, Murilo Pazin ${ }^{\mathrm{a}}$, Mariana Furio Franco-Bernardes ${ }^{\mathrm{a}}$, \\ Airton da Cunha Martins Jr ${ }^{\mathrm{a}}$, Gustavo Rafael Mazzaron Barcelos ${ }^{\mathrm{a}}$, Márcio Cesar Pereira ${ }^{\mathrm{c}}$, \\ João Paulo Mesquita ${ }^{c}$, Jairo Lisboa Rodrigues ${ }^{c}$, Fernando Barbosa $\mathrm{Jr}^{\mathrm{a}}$, Daniel Junqueira Dorta ${ }^{\mathrm{d}, \mathrm{e}}$ \\ ${ }^{\text {a }}$ Faculdade de Ciências Farmacêuticas de Ribeirão Preto, Departamento de Análises Clínicas, Toxicológicas e Bromatológicas, Universidade de São Paulo, Av. \\ Bandeirantes, 3900, Bairro Monte Alegre, CEP:14040901, Ribeirão Preto, São Paulo, Brazil \\ b Departamento Bioprocessos e Biotecnologia, Faculdade de Ciências Agronômicas, Fazenda Experimental de Lageado, Universidade Estadual Paulista “Júlio de Mesquita \\ Filho", Botucatu, SP, Brazil \\ ${ }^{\mathrm{c}}$ Instituto de Ciência, Engenharia e Tecnologia, Universidade Federal dos Vales do Jequitinhonha e Mucuri, CEP: 39803-371, Teófilo Otoni, Minas Gerais, Brazil \\ ${ }^{\mathrm{d}}$ Faculdade de Filosofia, Ciências e Letras de Ribeirão Preto, Departamento de Química, Universidade de São Paulo, Avenida do Café s/n', CEP 14040-903, Ribeirão \\ Preto, São Paulo, Brazil \\ ${ }^{\mathrm{e}}$ National Institute for Alternative Technologies of Detection, Toxicological Evaluation and Removal of Micropollutants and Radioactives (INCT-DATREM), Unesp, \\ Institute of Chemistry, P.O. Box 355, 14800-900 Araraquara, SP, Brazil
}

\section{A R T I C L E I N F O}

\section{Keyword:}

Nanomaterials

Silver nanoparticles

Titanium nanoparticles

Mitotoxicants

\begin{abstract}
A B S T R A C T
Nanotechnology is a growing branch of science that deals with the development of structural features bearing at least one dimension in the nano range. More specifically, nanomaterials are defined as objects with dimensions that range from 1 to $100 \mathrm{~nm}$, which give rise to interesting properties. In particular, silver and titanium nanoparticles (AgNPs and TiNPs, respectively) are known for their biological and biomedical properties and are often used in consumer products such as cosmetics, food additives, kitchen utensils, and toys. This situation has increased environmental and occupational exposure to AgNPs and TiNPs, which has placed demand for the risk assessment of NPs. Indeed, the same properties that make nanomaterials so attractive could also prove deleterious to biological systems. Of particular concern is the effect of NPs on mitochondria because these organelles play an essential role in cellular homeostasis. In this scenario, this work aimed to study how AgNPs and TiNPs interact with the mitochondrial respiration chain and to analyze how this interaction interferes in the bioenergetics and oxidative state of the organelles after sub-chronic exposure. Mitochondria were exposed to the NPs by gavage treatment for 21 days to check whether co-exposure of the organelles to the two types of NPs elicited any mitochondrion-NP interaction. More specifically, male Wistar rats were randomly assigned to four groups. Groups I, II, III, and IV received mineral oil, TiNPs $(100 \mu \mathrm{g} / \mathrm{kg} / \mathrm{day})$, AgNPs $(100 \mu \mathrm{g} / \mathrm{kg} / \mathrm{day})$, and TiNPs + AgNPs $(100 \mu \mathrm{g} / \mathrm{kg} /$ day $)$, respectively, by gavage. The liver was immediately removed, and the mitochondria were isolated and used within $3 \mathrm{~h}$. Exposure of mitochondria to TiNPs + AgNPs lowered the respiratory control ratio, causing an uncoupling effect in the oxidative phosphorylation system. Moreover, both types of NPs induced mitochondrial swelling. Extended exposure of mitochondria to the NPs maintained increased ROS levels and depleted the endogenous antioxidant system. The AgNPs and TiNPs acted synergistically-the intensity of the toxic effect on the mitochondrial redox state was more significant in the presence of both types of NPs. These findings imply that the action of the NPs on mitochondria underlie NP toxicity, so future application of NPs requires special attention.
\end{abstract}

\section{Introduction}

Nanotechnology is a growing process in domestic and industrial in recent years, and is dedicated to the development of materials and particles of structural characteristics with at least one dimension in the nano range. The International Organization for Standardization (ISO) has defined "nanomaterial" as a "material with any external dimension in the nanoscale or having internal structure or surface structure in the

\footnotetext{
* Corresponding author at: Faculdade de Ciências Farmacêuticas de Ribeirão Preto, Departamento de Análises Clínicas, Toxicológicas e Bromatológicas, Universidade de São Paulo, Av. Bandeirantes, 3900, Bairro Monte Alegre, CEP:14040901, Ribeirão Preto, São Paulo, Brazil.

E-mail address: liliancp@fca.unesp.br (L.C. Pereira).
} 
nanoscale" and "nanoparticle" as a "nano-object with all three external dimensions in the nanoscale" where nanoscale is defined as the size range from approximately 1-100 $\mathrm{nm}$ [1]. In particular, silver and titanium nanoparticles (AgNPs and TiNPs, respectively) are known for their biological and biomedical properties [2,3]. AgNPs display antimicrobial action and are potentially useful in the areas of food preservation, antibacterial surfaces and textiles, nanomedicine, and dentistry [2,4]. In turn, TiNPs have proven to be useful in cancer photodynamic therapy, drug delivery systems, cell imaging, biosensors for biological assay, and genetic engineering [3], not to mention that they provide interesting products such as opaque plastics [5]. Additionally, AgNPs and TiNPs are often present in consumer products such as cosmetics, food additives, kitchen utensils, and toys [6-8].

Increased environmental and occupational exposure to AgNPs and TiNPs has placed demand for the risk assessment of nanoparticles (NPs) [9]. NPs may penetrate the organism by oral, dermal contact or by inhalation $[7,10,11]$. Therefore, the same properties that make nanomaterials so attractive could also prove deleterious to biological systems [12] because the small size and large surface area of NPs could increase their toxicity as compared with the parent micrometer-sized particles $[4,13]$. Both, the manufacturing processes of AgNPs and their use increase the possibility of human exposure, basically through the gastrointestinal tract, through contaminated water and food [14]. While, TiNPs for example, can enter the human body through different routes such as inhalation (respiratory tract), ingestion, dermal penetration and injection [15]. In addition, TiNPs is among the nanoparticles most produced. However, AgNPs are the most popular advertised and together, silver and $\mathrm{TiO}_{2}$ are the NMs most combined with other NMs in the various consumer products containing NPs [8].

One of the main concerns about the toxicity of nanomaterials is that they can produce oxidative stress. NPs can also be transported across cell membranes, especially into mitochondria [16]. Besides being able to accumulate in hepatic cells, for example and mitochondria $[17,18]$. Although it is unclear whether nanomaterials target the mitochondria directly or act secondary to oxidative stress, they can damage the organelle $[12,19]$.

Mitochondria have important functions in cellular bioenergetics-they distribute the energy they produce throughout the cell, thereby playing an essential role in cellular homeostasis. Some of the mitochondrial metabolic pathways trigger the electron transport chain and oxidative phosphorylation, to generate ATP [20]. However, these processes also produce reactive oxygen species (ROS). Continuous ROS generation and elimination in biological systems help to regulate cellsignaling processes, but excess ROS culminate in oxidative stress and cell damage [21].

To protect the cell against oxidative stress, mitochondrial and cytosolic enzymes as well as non-enzymatic antioxidants, such as glutathione (GSH), scavenge ROS $[20,22]$. Depletion of these cellular antioxidants and increased ROS production due to the presence of xenobiotics, like nanomaterials, lead to oxidative stress, thereby damaging DNA, lipids, and proteins [23].

Besides raising ROS production, NPs can reduce the mitochondrial membrane potential and elicit a cascade of cytotoxic events, such as damage to the mitochondrial inner membrane, to cause cell death [23]. Hence, investigating whether NPs induce mitochondrial swelling can indicate if the permeability transition pores open. Opening of these pores reflects the loss of organelle ability to maintain ion and solute gradients across the inner membrane, which culminates in bioenergetic deficit and cell death [12].

Bearing the wide application of nanomaterials in mind, this work aimed to study how AgNPs and TiNPs interact with the mitochondrial respiration chain and to analyze how this interaction interferes in the bioenergetics and oxidative state of the organelle after sub-chronic exposure to the NPs. This work also examined the toxic effects of coexposure of mitochondria to AgNPs and TiNPs. With regard to the constitution of the NPs mixture, we hypothesized that the combined effects will lead to synergistic interactions between the compounds, where the toxic effects caused by mixture will exceed the sum of the separated NPs, and aimed to bring new insights to NPs toxicity. So the study aimed to bring new knowledge about ecotoxicological risk assessment of these environmental pollutants.

\section{Materials and methods}

\subsection{Chemicals}

Silver (CAS No. 7440-22-4) and titanium dioxide (CAS No 1346367-7) nanoparticles were obtained from Sigma-Aldrich (St. Louis, MO, USA). According to the supplier the Silver nanoparticles have ${ }^{<} 100 \mathrm{~nm}$ of particle size and $5.0 \mathrm{~m}^{2} / \mathrm{g}$ of surface area. While, titanium dioxide nanoparticles have $21 \mathrm{~nm}$ of particle size and $35-65 \mathrm{~m}^{2} / \mathrm{g}$ of surface area. Solutions of dispersed titanium and silver particles were prepared with mineral oil, which was used as the control vehicle. The reagents rotenone, carbonyl cyanide 3-chlorophenylhydrazone (CCCP), glutamate, malate, adenosine 5-diphosphate (sodium salt) (ADP), safranin-o, $o$-phthalaldehyde (OPT), ethylene glycol bis( $\beta$-aminoethyl ether)$\mathrm{N}, \mathrm{N}, \mathrm{N}, \mathrm{N}$-tetraacetic acid (EGTA), and tert-butyl hydroperoxide solution (t-BOOH) were purchased from Sigma-Aldrich Chemical Co (St Louis, MO, USA). The reagent 2,7-dichlorodihydrofluorescein diacetate $\left(\mathrm{H}_{2}\right.$ DCFDA) was obtained from Molecular Probes (OR, USA). All the other reagents were of the highest commercial degree. All the stock solutions were prepared with glass-distilled deionized water.

\subsection{Characterization}

Dynamic light scattering (DLS) measurements were performed with a Zetasizer Nano ZS90 DLS equipment (Malvern Instruments Ltd., England) to obtain information about the average hydrodynamic radius and the size distribution of the particles. A cuvette QS $3 \mathrm{~mm}$ was used as a sample container. Surface charge of nanoparticles was judged by zeta potential measurement on a Malvern Zetasizer 2000 HS (Malvern, UK).

The crystalline phases of the nanoparticles were determined using an X-ray diffractometer (XRD 6000, Shimadzu). The data were collected from 20 to $80^{\circ} 2 \theta$ at a step width of $0.5^{\circ}, 10 \mathrm{~s}$ per step, at $40 \mathrm{kV}$, $200 \mathrm{~mA}$, and CuKa radiation $(\lambda=1.540560 \AA)$. Silicon was used as an external standard.

\subsection{Animals}

Male Wistar rats weighing 180-200 g were used. The Committee for Experimental Animal Care and Use of the University of Sao Paulo, Brazil, approved all the experimental procedures (number 14.1.763.53.0). Animals were kept under a $12 \mathrm{~h}$ light:dark cycle, at an ambient temperature of $24 \pm 2{ }^{\circ} \mathrm{C}$, with free access to food and water.

\subsection{Treatments}

Male Wistar rats were randomly assigned to four groups of seven rats each. Groups I, II, III, and IV received mineral oil, TiNPs $(100 \mu \mathrm{g} /$ $\mathrm{kg} /$ day), AgNPs $(100 \mu \mathrm{g} / \mathrm{kg} /$ day $)$, and TiNPs + AgNPs $(100 \mu \mathrm{g} / \mathrm{kg} /$ day), respectively, by gavage. The total treatment time was 21 days, and the compounds were administered on a daily basis. After treatment, the animals were euthanized by applying an overdose of ketamine and xylazine, which was followed by collection of liver.

\subsection{Mitochondria isolation and performed assays}

After immediate removal of the rat liver (10-15 g), this organ was sliced into $50 \mathrm{~mL}$ of medium containing $250 \mathrm{mM}$ sucrose, $1 \mathrm{mM}$ EGTA, and $10 \mathrm{mM}$ HEPES-KOH, $\mathrm{pH} 7.2$, and homogenized three times in a Potter-Elvehjem homogenizer for $15 \mathrm{~s}$, at $1 \mathrm{~min}$ intervals. Rat liver mitochondria were isolated by standard differential centrifugation 
[24]. Homogenates were centrifuged at $580 \mathrm{~g}$ for $5 \mathrm{~min}$; the supernatant was further centrifuged at $10,300 \mathrm{~g}$ for $10 \mathrm{~min}$. The pellets were suspended in $10 \mathrm{~mL}$ of medium containing $250 \mathrm{mM}$ sucrose, $0.3 \mathrm{mM}$ EGTA, and $10 \mathrm{mM}$ HEPES-KOH, $\mathrm{pH} 7.2$, and centrifuged at $3400 \mathrm{~g}$ for $15 \mathrm{~min}$. The final mitochondrial pellet was suspended in $1 \mathrm{~mL}$ of medium con-

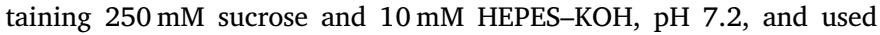
within $3 \mathrm{~h}$. All the procedures were carried out at $4{ }^{\circ} \mathrm{C}$, and the mitochondrial protein content was determined by the biuret reaction.

2.5.1 Mitochondrial swelling was estimated from the decrease in apparent turbidity at $540 \mathrm{~nm}$ measured with a Model DU-70 spectrophotometer (Beckman, Coulter Inc., Fullerton, CA, USA). The mitochondrial suspension $(0.4 \mathrm{mg}$ protein $/ \mathrm{mL})$ was incubated with the standard reaction medium plus $2.5 \mu \mathrm{M} \mathrm{Ca}^{2+}, 2.5 \mu \mathrm{M}$ rotenone, and $5 \mathrm{mM} / \mathrm{L}$ succinate, at $30^{\circ} \mathrm{C}$. Kinetics was evaluated for $10 \mathrm{~min}$ [25]. Inorganic phosphate was used as positive control for mitochondrial (just for information, without necessarily showing the data).

2.5.2 Mitochondrial respiratory rate was monitored on an oxygraph (Hansatech, Norfolk, England) equipped with a Clark-type oxygen electrode [26,27]. The mitochondria ( $1 \mathrm{mg}$ of protein/mL) were incubated in $1 \mathrm{~mL}$ of standard medium containing $125 \mathrm{mM}$ sucrose, $65 \mathrm{mM} \mathrm{KCl}, 10 \mathrm{mM}$ HEPES-KOH, $0.5 \mathrm{mM}$ EGTA, and $10 \mathrm{mM} \mathrm{K}_{2} \mathrm{HPO}_{4}$, $\mathrm{pH} 7.2$, at $30^{\circ} \mathrm{C}$. Glutamate and malate at $5 \mathrm{mM}$ were used as the oxidizable substrates for complex I of the respiratory chain. The phosphorylation was induced with $400 \mathrm{nmol}$ of ADP for all assays.

2.5.3 Mitochondrial reactive oxygen species and reactive nitrogen species (ROS/RNS) production/accumulation was monitored on a spectrofluorometer by using $5 \mu \mathrm{M} 22^{\prime}, 7^{\prime}$-dichlorodihydrofluorescein diacetate $\left(\mathrm{H}_{2} \mathrm{DCFDA}\right)$ [27] as probe and the $503 / 529 \mathrm{~nm}$ excitation/emission wavelength pair. The mitochondria ( $1 \mathrm{mg}$ of protein $/ \mathrm{mL}$ ) were incubated in $2 \mathrm{~mL}$ of the standard reaction medium containing $125 \mathrm{mM}$ sucrose, $65 \mathrm{mM} \mathrm{KCl}, 10 \mathrm{mM}$ HEPES-KOH, $2.5 \mu \mathrm{M}$ rotenone, and $5 \mathrm{mM}$ succinate at $30^{\circ} \mathrm{C}$. The kinetic evaluation lasted $10 \mathrm{~min}$.

2.5.4 Determination of the GSH/GSSG ratio. A mitochondrial suspension ( $1 \mathrm{mg}$ of protein $/ \mathrm{mL}$ ) was treated with $0.5 \mathrm{~mL}$ of $13 \%$ trichloroacetic acid and centrifuged at $900 \mathrm{~g}$ for $3 \mathrm{~min}$. To determine GSH, aliquots $(100 \mu \mathrm{L})$ of the supernatant were mixed with $2 \mathrm{~mL}$ of $100 \mathrm{mM}$ $\mathrm{NaH}_{2} \mathrm{PO} 4$ buffer, pH 8.0, containing 5 mM EGTA. Next, $100 \mu \mathrm{L}$ ophthaldialdehyde $(1 \mathrm{mg} / \mathrm{mL})$ was added, and fluorescence was measured 15 min later by using the $350 / 420 \mathrm{~nm}$ excitation/emission wavelength pair [28]. GSSG was determined by the addition of aliquots $(250 \mu \mathrm{L})$ of the supernatant to $250 \mu \mathrm{L}$ of $0.04 \mathrm{M} \mathrm{N}$-ethylmaleimide. After $20 \mathrm{~min}$ of incubation at room temperature, $500 \mu \mathrm{L}$ of $1 \mathrm{M} \mathrm{NaOH}$ was added. Then, $100 \mu \mathrm{L}$ of the resulting solution was added to $2 \mathrm{~mL}$ of $1 \mathrm{M} \mathrm{NaOH}$ and $100 \mu \mathrm{L}$ of o-phthaldialdehyde $(1 \mathrm{mg} / \mathrm{mL})$. The fluorescence was measured $15 \mathrm{~min}$ later in a model F-4500 Hitachi fluorescence spectrophotometer by using the $350 / 420 \mathrm{~nm}$ excitation/emission wavelength pair [28].

\subsection{Statistical analysis}

The results were evaluated by analysis of variance (ANOVA), followed by Dunnet test for comparison of the several treated groups with the control group; the program GraphPrism, version 5.1 for Windows was used. Results with $\mathrm{p} \leq 0.05$ were considered statistically significant.

\section{Results}

The qualitative analyses of the XRD patterns of the nanoparticles indicated that the sample TiNP is constituted of anatase (JCPDS 1-562) and rutile (JCPDS 1-1292). The sample AgNP is formed by a single phase due to the metal silver (JCPDS 4-783). The mean size of AgNP crystallites were $<100 \mathrm{~nm}$ with a surface area of $5.0 \mathrm{~m}^{2} / \mathrm{g}$ and for TiNP the mean size were $21 \mathrm{~nm}$ and the surface area was $35-65 \mathrm{~m}^{2} / \mathrm{g}$. The zeta potential of the TiNP and AgNP were $-5.07 \pm 1.11$ and $-5.73 \pm 1.23$, respectively. These low zeta potential values indicate

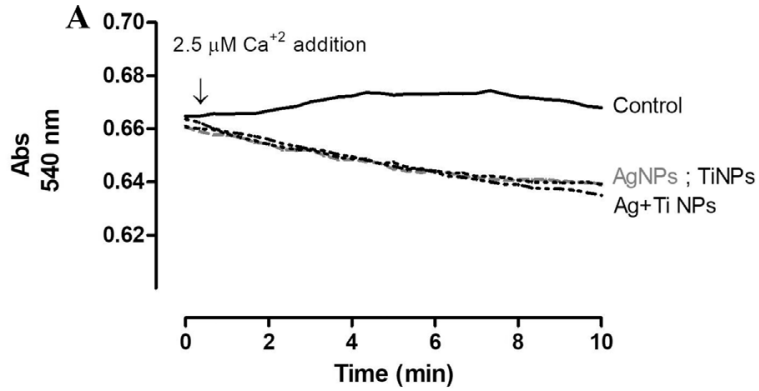

B

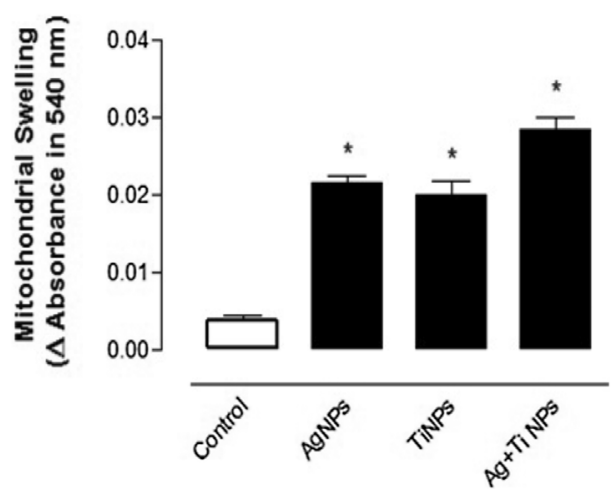

Fig. 1. Mitochondrial swelling monitored with the change of absorbance at $540 \mathrm{~nm}$ (A540) of the mitochondrial suspension over $10 \mathrm{~min}$. The arrow indicates addition of $2.5 \mu \mathrm{M} \mathrm{CaCl}{ }_{2}$. The traces represent typical direct recordings of experiments performed for each group. Control $=$ mineral oil; AgNPs $=$ Silver nanoparticles $(100 \mu \mathrm{g} / \mathrm{kg} / \mathrm{day})$; TiNPs = Titanium Nanoparticles $(100 \mu \mathrm{g} / \mathrm{kg} /$ day $)$; and AgNPs + TiNPs = Co-exposure to Silver and Titanium Nanoparticles $(100 \mu \mathrm{g} / \mathrm{kg} /$ day $)$. Data represent the mean + SEM of different mitochondrial preparations. *Indicates statistically significant difference versus control $(\mathrm{p}<0.05)$.

that the particles are poorly charged and attract each other due to the low particle stabilization by the surfactant, which results in agglomeration of particles.

Mitochondrial swelling accounts for the release of proteins located in the matrix or in the intermembrane space of mitochondria exposed to xenobiotics [29,30]. Fig. 1A shows how exposure to NPs induced mitochondrial swelling and $1 \mathrm{~B}$ shows the variation in absorbance observed during the monitored time. Both AgNPs and TiNPs induced mitochondrial swelling. However, concomitant administration of these NPs, to simulate co-exposure, shows synergistic effects with respect to mitochondrial swelling. In other words, when they were administered together, AgNPs and TiNPs induced swelling more extender as if they were administered alone.

To check the effect of exposing mitochondria to either AgNPs or TiNPs and to both types of NPs simultaneously on mitochondrial bioenergetics, we conducted a mitochondrial respiration assay using mix with glutamate + malate as oxidizable substrates that donate electron to complex I of the respiratory chain. Fig. 2A illustrates how AgNPs, TiNPs, and the association of these NPs affected glutamate and malate-supported respiration. The respiratory control ratio (RCR) decreased upon exposure of the mitochondria to the combination AgNPs + TiNPs, but not upon exposure of the organelles to AgNPs or TiNPs alone. The ADP consumption by oxygen molecule (ADP/O) diminished in the group treated with AgNPs alone and with the association AgNPs + TiNPs (Fig. 2B). In addition, State IV respiration significantly increased in the group treated with AgNPs + TiNPs (V4 $=13.26 \pm 0.08)$ as compared with the control group (V4 $=7.87 \pm 1.16)$, which indicated uncoupling of the mitochondria (Fig. 2C).

The presence of toxic compounds can impact the mitochondrial respiratory chain negatively-ROS production may increase, to 

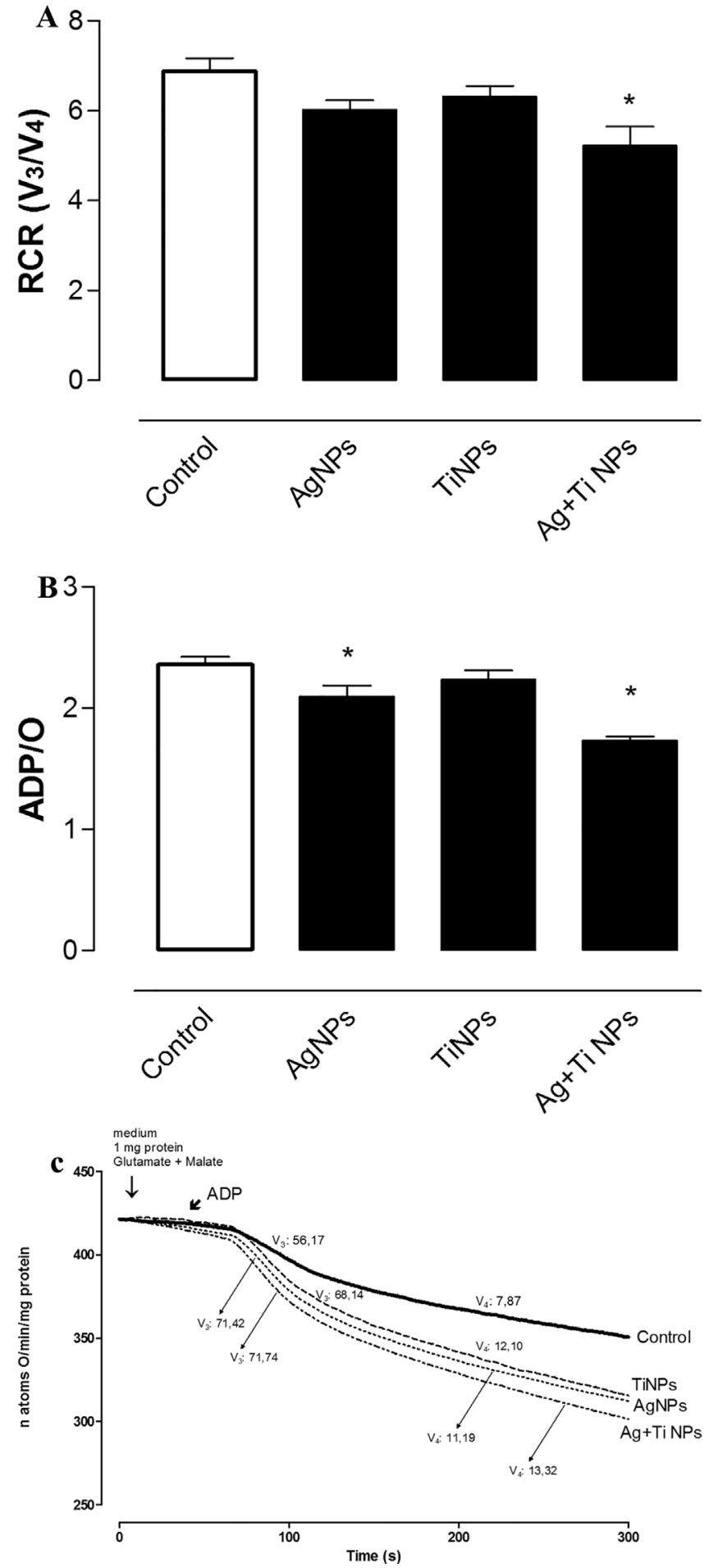

Fig. 2. Respiratory control ratio (RCR) in liver mitochondria (Fig. 2A), ADP/O (Fig. 2B) and their outline (Fig. 2C) upon incubation of mitochondria with nanoparticles. Data represent the mean + SEM of different mitochondrial preparations. *Indicates statistically significant difference versus control $(\mathrm{p}<0.05)$. Control $=$ mineral oil; AgNPs = Silver $\quad$ nanoparticles; $\quad$ TiNPs = Titanium $\quad$ Nanoparticles; $\quad$ and AgNPs + TiNPs $=$ Co-exposure to Silver and Titanium Nanoparticles.

culminate in mitochondrial and cellular damage by oxidative stress [31].

Knowing that many NPs can induce oxidative stress and therefore impact mitochondrial bioenergetics, we evaluated ROS and RNS accumulation in mitochondria in the presence of the target NPs. According to Fig. 3, ROS/RNS levels augmented in succinate-energized

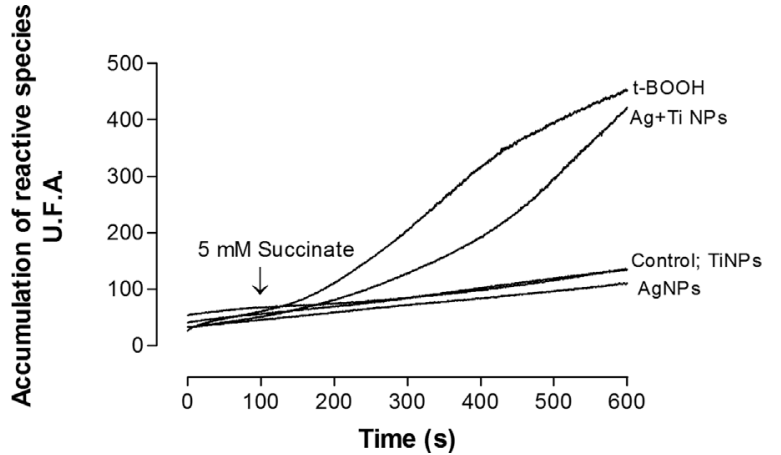

Fig. 3. Effect of exposure to nanoparticles on ROS accumulation as assessed with the fluorescence probe $2^{\prime}, 7^{\prime}$-dichlorodihydrofluorescein diacetate $\left(\mathrm{H}_{2} \mathrm{DCFDA}\right)$. The mitochondria were incubated in a standard reaction medium. Energization was achieved with $5 \mathrm{mM}$ Succinate. Data are presented as the mean + SEM of different mitochondrial preparations. *Significantly different $(\mathrm{p}<0.05)$ from the control (mineral oil). tert-butyl hydroperoxide (t-BOOH) $300 \mu \mathrm{M}$ was used to induce oxidative stress. Control $=$ mineral oil; $\quad$ AgNPs = Silver nanoparticles; $\quad$ TiNPs = Titanium Nanoparticles; and AgNPs + TiNPs $=$ Co-exposure to Silver and Titanium Nanoparticles.

mitochondria exposed to both NPs when treated concomitantly, as indicated by oxidation of the nonspecific probe $\mathrm{H}_{2}$ DCF-DA, a broadspectrum probe that aids detection of reactive species in general. In the case of oxidative stress, co-exposure of mitochondria to AgNPs + TiNPs promoted the amount of reactive species significantly increased in Group IV that an exposure of a nanoparticle alone was not able to present. To check how the resulting reactive species influenced the endogenous antioxidant system, we calculated the GSH/GSSG ratio (Fig. 4). Treatment of mitochondria with TiNPs and co-exposure of mitochondria to AgNPs + TiNPs modified the GSH/GSSG ratio, but AgNPs did not deplete the endogenous antioxidant systems.

\section{Discussion}

Nanotoxicology, or the study of the potential adverse effects of nanomaterials on the human body and the environment, has become increasingly important and will continue to attract researchers' attention for many years to come because an ever-growing number of consumer products based on nanomaterials are continuously entering the market [32-34]. The lack of data on the impact of human exposure to

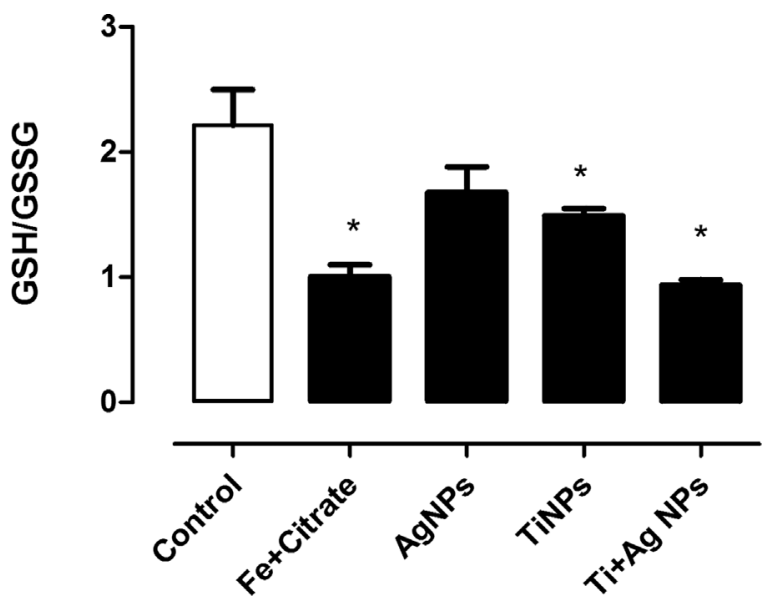

Fig. 4. Effects of exposure to nanoparticles on GSH/GSSG ratio as analyzed with the fluorescence probe $o$-phthalaldialdehyde (OPT). The mitochondria were incubated in a standard reaction medium. Energization was achieved with $5 \mathrm{mM}$ Succinate, carried out as described in Section 2. Data are presented as the mean + SEM of different mitochondrial preparations. *Significantly different $(\mathrm{p}<0.05)$ from the control (mineral oil). $50 \mu \mathrm{M}$ FeSO 4 plus $2 \mathrm{mM}$ sodium citrate ( $\mathrm{Fe}+$ Citrate) was used to induce oxidative stress. $\quad$ AgNPs $=$ Silver nanoparticles; $\quad$ TiNPs = Titanium Nanoparticles; and AgNPs + TiNPs $=$ Co-exposure to Silver and Titanium Nanoparticles. 
NPs in the workplace and on the release of NPs from consumer products into the environment makes risk assessment of these materials difficult [35]. NPs can reach the systemic circulation after inhalation, ingestion, or intravenous injection, followed by further distribution and accumulation of NPs in several organs such as lung, liver, spleen, kidneys, brain, and heart [36,37]. Recent evidence has shown that liver and bile ducts are targets of AgNP toxicity: $\mathrm{Ag}^{+}$accumulates in liver following exposure to AgNPs [38,39].

This study consisted in an attempt to identify the adverse effects of AgNPs, TiNPs and co-exposure to both, by using mitochondria isolated from rat liver. Our study demonstrated that AgNPs and TiNPs affected mitochondrial bioenergetics and co-exposure can induce oxidative stress, as evidenced by reactive species production and accumulation and deregulation of the endogenous antioxidant system.

In general, NPs and derivatives have been described to cause multiple effects on mitochondria, ultimately leading to hepatic cell death [40-42]. Although the present study involved isolated mitochondria, the results denoted the specific potential action that AgNPs and TiNPs have on this target organelle. The results also corroborated with reports that exposure to NPs can trigger cell death because mitochondria is intimately linked with regulation and signaling of cell death pathways $[29,43,44]$. Our results corroborate and may help explain the findings of Miranda et al. [45], these researchers observed that exposure to AgNPs resulted in hepatotoxicity, inducing cell death by apoptosis. Once we observed mitochondrial dysfunction in hepatic mitochondria, we suggest that there was the activation of the intrinsic apoptosis pathway [45].

Mitochondrial permeability transition pores (MPTPs) are related to calcium accumulation inside the mitochondria and to rise in oxygen free radicals [46]. Opening of these pores can lead to uncoupling of mitochondria and to massive matrix destruction [30]. If the pores remain open, cells cannot maintain their ATP levels, and cell death occurs. All the exposure conditions evaluated herein caused swelling of the organelle, a process that is closely related to MPTP opening [47]. This effect is consistent with literature results showing that NPs can induce mitochondrial dysfunctions after swelling [12,33].

The intracellular ATP content reflects the state of mitochondria. ATP depletion, a marked event of compound toxicity, can originate from impaired processes in mitochondria such as inhibition of electron transport in the mitochondrial membrane, membrane potential dissipation, and generation of reactive species [44,48]. Lower oxidative phosphorylation efficiency, as judged from the ADP/O rate and RCR ratio, added to mitochondrial swelling and induced oxidative stress, were in line with other works reporting that the amount of ATP in A549 lung cells exposed to TiNPs decreased [33].

The mitochondria of AgNP-treated rats presented less efficient oxidative phosphorylation and lower ADP/O rate. This effect was even more pronounced in the mitochondria of rats treated with AgNPs + TiNPs (Fig. 2B). Teodoro et al. [11] also exposed mitochondria to NPs during the time of the in vitro assays and verified altered mitochondrial bioenergetics in the presence of AgNPs. Like us, these researchers [12] detected smaller RCR, MPTP opening, and mitochondrial swelling related to uncoupling of mitochondria. Another study showed that exposure to TiNPs also reduced RCR in lung mitochondria [49]. In the present study, this effect clearly took place in vivo upon exposure of the animals to both AgNPs and TiNPs. Taken together, these results indicated loss of oxidative phosphorylation capacity, justified by increased oxygen consumption in State IV of mitochondrial respiration.

Adaptive or maladaptive responses can activate MPTP and cause intra- and intermitochondrial redox environment changes [50]. Free radical production bears direct correlation with NP cytotoxicity both in vivo and in vitro [51-53]. Oxidative damage of cellular constituents by ROS generation or inactivation of the antioxidant defense system probably induces NP cytotoxicity [54]. Consequently, NPs can induce DNA damage and apoptosis through ROS generation and oxidative stress $[55,56]$, leading to cellular damage, DNA adducts, and genotoxicity $[57,58]$. Indeed, genotoxic effects have already been observed in Human Fibroblasts Cells exposed to AgNPs [55].

Based on the results of this study, co-exposure to AgNPs and TiNPs may have induced considerable hepatic oxidative stress after oral exposure, as judged from increased reactive species formation and reduced activity of the endogenous antioxidant system represented by the GSH/GSSG ratio. The Glutathione S-Transferase (GST) enzymes together with the endogenous tripeptide glutathione (GSH) can operate to limit ROS-induced injury in organelles, cells, and tissues by maintaining the intracellular redox balance [59-61].

Concomitant administration of AgNPs and TiNPs decreased the redox state of the endogenous antioxidant system, represented by the GSH/GSSG ratio, as a result of the increased reactive species production measured by the DCF probe. In these cases, GSH did not kept the liver tissue of animals treated with NPs free of oxidative stress. Furthermore, co-exposure of rat liver to both AgNPs and TiNPs may have promoted a synergistic effect, as attested by the higher intensity of the toxic effect on the mitochondrial redox state in the presence of both types of NPs.

NPs induce mitochondrial damage via ROS production. Treatment of a rat liver cell line (BRL 3A cells) with AgNPs resulted in reduced GSH levels and a $25-\mu \mathrm{g} / \mathrm{mL}$ increase in ROS [62]. Treatment of mitochondria from lung tissue with $\mathrm{TiO}_{2} \mathrm{NPs}$ augmented ROS by $46 \%$, but it was not enough to modify important enzymatic and nonenzymatic antioxidants and lipid peroxidation of the mitochondrial protein after exposure to the NPs for one hour [49]. However, the present work demonstrated that extended exposure with concomitant administration of AgNPs and TiNPs (for 21 days by gavage treatment) was sufficient to maintain increased ROS levels and deplete the endogenous antioxidant system.

In line with these observations, accumulation studies showed that NPs can harm various animal organs, including lung, liver, spleen, and kidney [63], and induction of oxidative stress after exposure to different NPs was the main mechanism of toxicity [64-66]. Additionally, antioxidant enzyme levels changed significantly, and other literature works have reported altered antioxidant system in animals and plants exposed to NPs $[41,66,67]$. Many studies have been reported adverse effects observed for AgNP, for example, in mammalian cells in the $\mathrm{mg}$ / $\mathrm{kg}$ /day range. In this work we present toxic effects with exposure 100 times lower $(\mu \mathrm{g} / \mathrm{kg} /$ day $)$.

In conclusion, the growing popularity and increasing use of NPs has aroused the interest in nanotoxicology. This work has described a small portion of the toxicity of NPs and NP associations. Gathering all results we can conclude that in decreasing index of toxicity we have $\mathrm{Ag}+$ TiNPs higher than TiNPs that in turn is larger than AgNPs. Our results should assist lawmakers to pose limits to the use of NPs in commercial products and issue new regulations. The adverse effects of human exposure to NPs involve mitochondria therefore require special attention.

\section{Conflict of interest}

The authors declare that there is no conflict of interests regarding the publication of this manuscript.

\section{Acknowledgments}

Authors thank the São Paulo Research Foundation, the National Council for Technological and Scientific Development (CNPq) and Coordination for the Improvement of Higher Education Personnel (CAPES) for financial support.

\section{References}

[1] E. Frohlich, Cellular targets and mechanisms in the cytotoxic action of non-biodegradable engineered nanoparticles, Curr. Drug Metab. 14 (2013) 976-988. 
[2] R.P. Singh, P. Ramarao, Cellular uptake: intracellular trafficking and cytotoxicity of silver nanoparticles, Toxicol. Lett. 213 (2012) 249-259.

[3] Z.F. Yin, L. Wu, H.G. Yang, Y.H. Su, Recent progress in biomedical applications of titanium dioxide, Phys. Chem. Chem. Phys. 15 (2013) 4844-4858.

[4] M. Moritz, M. Geszke-Moritz, The newest achievements in synthesis: immobilization and practical applications of antibacterial nanoparticles, Chem. Eng. J. 228 (2013) 596-613.

[5] X. Huang, M.J. Bolen, N.S. Zacharia, Silver Nanoparticle aided self-healing of polyelectrolyte multilayers, Phys. Chem. Chem. Phys. 22 (2014) 10267-10273, http://dx.doi.org/10.1039/c4cp0034g.

[6] R. Behra, L. Sigg, M.J. Clift, F. Herzog, M. Minghetti, B. Johnston, A. Petri-Fink, B. Rothen-Rutishauser, Bioavailability of silver nanoparticles and ions: from a chemical and biochemical perspective, J. R. Soc. Interface. 10 (2013) 1-15, http:// dx.doi.org/10.1098/rsif.2013.0396.

[7] R. Tassinari, F. Cubadda, G. Moracci, F. Aureli, M. D’Amato, M. Valeri, B. de Berardis, A. Raggi, A. Mantovani, D. Passeri, M. Rossi, F. Maranghi, Oral, short-term exposure to titanium dioxide nanoparticles in Sprague-Dawley rat: focus on reproductive and endocrine systems and spleen, Nanotoxicology 25 (2013) 654-662.

[8] M.E. Vance, T. Kuiken, E.P. Vejerano, S.P. McGinnis, M.F. Hochella, D. Rejeski, M.S. Hull, Nanotechnology in the real world: redeveloping the nanomaterial consumer products inventory, Beilstein J. Nanotechnol. 6 (2016) 1769-1780.

[9] N.C. Mueller, B. Nowack, Exposure modeling of engineered nanoparticles in the environment, Environ. Sci. Technol. 42 (2008) 4447-4453.

[10] J. Wang, G. Zhou, C. Chen, H. Yu, T. Wang, Y. Ma, G. Jia, Y. Gao, B. Li, J. Sun, Y. Li, F. Jiao, Y. Zhao, Z. Chai, Acute toxicity and biodistribution of different sized titanium dioxide particles in mice after oral administration, Toxicol. Lett. 168 (2007) $176-185$.

[11] M. Crosera, M. Bovenzi, G. Maina, G. Adami, C. Zanette, C. Florio, F. Filon Larese, Nanoparticle dermal absorption and toxicity: a review of the literature, Int. Arch. Occup. Environ. Health 82 (2009) 1043-1055.

[12] J.S. Teodoro, A.M. Simões, F.V. Duarte, A.P. Rolo, R.C. Murdoch, S.M. Hussain, C.M. Palmeira, Assessment of the toxicity of silver nanoparticles in vitro: a mitochondrial perspective, Toxicol. In Vitro 25 (2011) 664-670.

[13] M.F. Song, Y.S. Li, H. Kasai, K. Kawai, Metal nanoparticle-induced micronuclei and oxidative DNA damage in mice, J. Clin. Biochem. Nutr. 50 (2012) 211-216.

[14] L. Vila, R. Marcos, A. Hernández, Long-term effects of silver nanoparticles in caco-2 cells, Nanotoxicology 11 (2017) 771-780.

[15] S.C. Liu, L.J. Xu, T. Zhang, G.G. Ren, Z. Yang, Oxidative stress and apoptosis induced by nanosized titanium dioxide in PC12 cells, Toxicology 267 (2010) $172-177$.

[16] S. Foley, C. Crowley, M. Smaihi, C. Bonfils, B. Erlanger, P. Seta, C. Larroque, Cellular localisation of a water-soluble fullerene derivative, Biochem. Biophys. Res. Commun. 294 (2002) 116-119.

[17] X. Sun, Z. Wang, S. Zhai, Y. Cheng, J. Liu, B. Liu, In vitro cytotoxicity of silver nanoparticles in primary rat hepatic stellate cells, Mol. Med. Rep. 8 (2013) $1365-1372$.

[18] J.S. Teodoro, R. Silva, A.T. Varela, F.V. Duarte, A.P. Rolo, S. Hussain, C.M. Palmeira, Low-dose, subchronic exposure to silver nanoparticles causes mitochondrial alterations in Sprague-Dawley rats, Nanomedicine 11 (2016) $1359-1375$.

[19] N. Li, C. Sioutas, A. Cho, D. Schmitz, C. Misra, J. Sempf, M. Wang, T. Oberley, J. Froines, A. Nel, Ultrafine particulate pollutants induce oxidative stress and mitochondrial damage, Environ. Health Perspect. 111 (2003) 455-460.

[20] A. Heller, G. Brockhoff, A. Goepferich, Targeting drugs to mitochondria, Eur. J Pharm. Biopharm. 82 (2012) 1-18.

[21] M.J. Piao, K.A. Kang, I.K. Lee, H.S. Kim, S. Kim, J.Y. Choi, J. Choi, J.W. Hyuna, Silver nanoparticles induce oxidative cell damage in human liver cells through inhibition of reduced glutathione and induction of mitochondria-involved apoptosis, Toxicol. Lett. 201 (2011) 92-100.

[22] B.V. Chernyak, O.Y. Pletjushkina, D.S. Izyumov, K.G. Lyamzaev, A.V. Avetisyan, Bioenergetics and death, Biochemistry 70 (2005) 240-245.

[23] Y.N. Wu, L.X. Yang, X.Y. Shi, I.C. Li, J.M. Biazik, R. Kyle, K.R. Ratinac, D.H. Chen, P. Thordarson, D.B. Shieh, F. Braet, The selective growth inhibition of oral cancer by iron core-gold shell nanoparticles through mitochondria-mediated autophagy, Biomaterials 32 (2011) 4565-4573.

[24] P.L. Pedersen, J.W. Greenawalt, B. Reynafarje, J. Hullihen, G.L. Decker, J.W. Soper, E. Bustamante, Preparation and characterization of mitochondria and submitochondrial particles of rat liver and liver derived tissues, Methods Cell Biol. 20 (1978) 411-481.

[25] M. Zoratti, I. Szabo, The mitochondrial permeability transition, Biochimica et Biophysica Acta (BBA) - Rev. Biomembr. 1241 (1995) 139-176.

[26] B. Chance, G.R. Willians, The respiratory chain and oxidative phosphorylation, Adv. Enzymol. Relat. Subj. Biochem. 17 (1956) 65-134.

[27] M. Wrona, K.B. Patel, P. Wardman, Reactivity of 2',7'-dichlorodihydrofluorescein and dihydrorhodamine 123 and their oxidized forms towards carbonate, nitrogen dioxide, and hydroxyl radicals, Free Radic. Biol. Med. 38 (2005) 262-270.

[28] P.J. Hissin, A. Hilf, A fluorimetric method for determination of oxidized and reduced glutathione in tissues, Anal. Biochem. 74 (1976) 214-226.

[29] A. Rasola, P. Bernardi, The mitochondrial permeability transition pore and its involvement in cell death and in disease pathogenesis, Apoptosis 12 (2007) 815-833.

[30] A.P. Halestrap, What is the mitochondrial permeability transition pore, J. Mol. Cell. Cardiol. 46 (2009) 821-831.

[31] B. Halliwell, J.M.C. Gutteridge, Free Radicals in Biology and Medicine, Third ed. Oxford University Press, New York, 1999.

[32] M. Ahamed, M.S. Alsalhi, M.K.J. Siddiqui, Silver nanoparticle applications and human health, Clin. Chim. Acta 411 (2010) 1841-1848.
[33] Y. Tang, F. Wang, C. Jin, H. Liang, X. Zhong, Y. Yang, Mitochondrial injury induced by nanosized titanium dioxide in A549 cells and rats, Environ. Toxicol. Pharmacol. 3 (6) (2013) 66-72.

[34] N. Singh, B. Manshian, G.J.S. Jenkins, S.M. Griffiths, P.M. Williams, T.G.G. Maffeis, C.J. Wright, S.H. Doak, Nano Genotoxicology The DNA damaging potential of engineered nanomaterials, Biomaterials 30 (2009) 3891-3914.

[35] Y.S. Kim, M.Y. Song, J.D. Park, K.S. Song, H.R. Ryu, Y.H. Chung, H.K. Chang, J.H. Lee, K.H. Oh, B.J. Kelman, I.K. Hwang, I.J. Yu, Subchronic oral toxicity of silver nanoparticles, Parti. Fibre Toxicol. 7 (2010) 2-11.

[36] A. Shimida, N. Kawamura, M. Okajima, T. Kaewamatawong, H. Inoue, T. Morita, Translocation pathway of the intra tracheally instilled ultrafine particles from the lung into blood circulation in the mouse, Toxicol. Pathol. 34 (2006) 949-957.

[37] A.C. Martins, L.F. Azevedo, C.C.S. Rocha, M.F.H. Carneiro, V.P. Venancio, M.R. Almeida, L.M.G. Antunes, R.C. HottJ, J.L. Rodrigues, A.T. Ogunjimi, J.A. Adeyemi, F. Barbosa, Evaluation of distribution redox parameters, and genotoxicity in Wistar rats co-exposed to silver and titanium dioxide nanoparticles, J. Toxicol. Environ. Health A 80 (2017) 1156-1165.

[38] J.H. Ji, J.H. Jung, S.S. Kim, J.U. Yoon, J.D. Park, B.S. Choi, Y.H. Chung, I.H. Kwon, J. Jeong, B.S. Han, J.H. Shin, J.H. Sung, K.S. Song, I.J. Yu, Twenty-eightday inhalation toxicity study of silver nanoparticles in Sprague-Dawley rats, Inhal. Toxicol. 19 (2007) 857-871.

[39] Y.S. Kim, J.S. Kim, H.S. Cho, D.S. Rha, J.M. Kim, J.D. Park, B.S. Choi, R. Lim, H.K. Chang, Y.H. Chung, I.H. Kwon, J. Jeong, B.S. Han, I.J. Yu, Twenty-eight-day oral toxicity genotoxicity, and gender-related tissue distribution of silver nanoparticles in Sprague-Dawley rats, Inhal. Toxicol. 20 (2008) 575-583.

[40] M.A. Siddiqui, H.A. Alhadlaq, J. Ahmad, A. Al-Khedhairy, J. Mussarat, M. Ahamed, Copper oxide nanoparticles induced mitochondria mediated apoptosis in human hepatocarcinoma cells, PLoS One 8 (2013), http://dx.doi.org/10.1371/journal pone.0069534.

[41] X. Lu, J. Qian, H. Zhou, Q. Gan, W. Tang, J. Lu, Y. Yuan, C. Liu, In vitro cytotoxicity and induction of apoptosis by silica nanoparticles in human HepG2 hepatoma cells, Int. J. Nanomed. 6 (2011) 1889-1901.

[42] M.S. Al-Qubaisi, A. Rasedee, M.H. Flaifel, S.H. Ahmad, S. Hussein-Al-Ali, M.Z. Hussein, Z. Zainal, F.H. Alhassan, Y.H. Taufig-Yap, E.E. Eid, I.A. Arbab, B.A. Al-Asbahi, T.J. Webster, M.E.E. Zowalaty, Induction of apoptosis in cancer cells by NiZn ferrite nanoparticles through mitochondrial cytochrome C release, Int. J. Nanomed. 8 (2013) 4115-4130.

[43] P. Bernardi, L. Scorrano, R. Colonna, V. Petronilli, F. Di Lisa, Mitochondria and cell death - mechanistic aspects and methodological issues, Eur. J. Biochem. 264 (1999) 687-701.

[44] K.B. Wallace, A.A. Starkov, Mitochondrial targets of drug toxicity, Annu. Rev. Pharmacol. Toxicol. 40 (2000) 353-388.

[45] R.R. Miranda, A.G. Bezerra Jr., C.A.O. Ribeiro, M.A.F. Randi, C.M. Voigt, L. Skytte, K.D. Rasmussen, F. Kjeldsen, F.F. Neto, Toxicological interactions of silver nanoparticles and non-essential metals in human hepatocarcinoma cell line, Toxicol. In Vitro 40 (2017) 34-143.

[46] A.P. Halestrap, Calcium: mitochondria and reperfusion injury: a pore way to die, Biochem. Soc. Trans. 34 (2006) 232-237.

[47] A.P. Halestrap, E. Doran, J.P. Gillespie, A. O'Toole, Mitochondria and cell death, Biochem. Soc. Trans. 28 (2000) 170-177.

[48] A.J. Kowaltowski, R.F. Castilho, A.E. Vercesi, Mitochondrial permeability transition and oxidative stress, FEBS Lett. 495 (2001) 12-15.

[49] V. Freyre-Fonseca, N.L. Delgado-Buenrostro, E.B. Gutiérrez-Cirlos, C.M. CalderónTorres, T. Cabellos-Avelar, Y. Sánchez-Pérez, E. Pinzón, I. Torres, E. Molina-Jijón, C. Zazueta, J. Pedraza-Chaverri, C.M. García-Cuéllar, Y.I. Chirino, Titanium Dioxide nanoparticles impair lung mitochondrial function, Toxicol. Lett. 202 (2011) 111-119.

[50] D.B. Zorov, M. Juhaszova, S. Sollott, Mitochondrial reactive oxygen species (ROS) and ros-induced ROS release, Phys. Rev. 94 (2014) 909-950.

[51] S. Lu, R. Duffin, C. Poland, P. Daly, F. Murphy, E. Drost, W. MacNee, V. Stone, K. Donaldson, Efficacy of simple short-term in vitro assays for predicting the potential of metal oxide nanoparticles to cause pulmonary inflammation, Environ. Health Perspect. 117 (2009) 241-247.

[52] E. Bressan, L. Ferroni, C. Gardin, C. Rigo, M. Stocchero, V. Vindigni, W. Cairns, B. Zavan, Silver nanoparticles and mitochondrial interactions, Int J Dent. (2013) 1-8.

[53] C. Greulich, J. Diendorf, T. Simon, G. Eggeler, M. Epple, M. Koller, Uptake and intracellular distribution of silver nanoparticles in human mesenchymal stem cells, Acta Biomater. 7 (201) (2016) 347-354.

[54] A. Nel, T. Xia, L. Madler, N. Li, Toxic potential of materials at the nano-level, Science 311 (2006) 622-627.

[55] P.V. AshaRani, L.K.M. Grace, M.P. Hande, S. Aliyaveettil, Cytotoxicity and genotoxicity of silver nanoparticles in human cells, ACS Nano 3 (2009) 279-290.

[56] E.J. Park, J. Choi, Y. Park, K. Park, Oxidative stress induced by cerium oxide nanoparticles in cultured BEAS-2B cells, Toxicology 1 (2008) 90-100.

[57] C. Carlson, S.M. Hussain, A.M. Schrand, L.K. Braydich-Stolle, K.L. Hess, R.L. Jones, J.J. Schlager, Unique cellular interaction of silver nanoparticles: sizedependent generation of reactive oxygen species, J. Phys. Chem. B 112 (2008) 13608-13619.

[58] Y. Li, D.H. Chen, J. Yan, Y. Chen, R.A. Mittelstaedt, Y. Zhang, A.S. Biris, R.H. Heflich, T. Chen, Genotoxicity of silver nanoparticles evaluated using the Ames test and in vitro micronucleus assay, Mutat. Res. 745 (2012) 4-10.

[59] I. Bousová, L. Skálová, Inhibition and induction of glutathione $S$-transferases by flavonoids: possible pharmacological and toxicological consequences, Drug Metab. Rev. 44 (2012) 267-286.

[60] B. Ketterer, B. Coles, D.J. Meyer, The role of glutathione in detoxication, Environ. Health Perspect. 49 (1983) 59-69. 
[61] F. Gu, V. Chauhan, A. Chauhan, Gluthathione redox imbalance in brain disorders, Curr. Opin. Clin. Nutr. Metab. Care 18 (2014) 89-95.

[62] S.M. Hussain, K.L. Hess, J.M. Gearhart, K.T. Geiss, J.J. Schlager, In vitro toxicity of nanoparticles in BRL 3A rat liver cells, Toxicol. In Vitro 19 (2005) 975-983.

[63] H.W. Chen, S.F. Su, C.T. Chien, W.H. Lin, S.L. Yu, C.C. Chou, J.J. Chen, P.C. Yang, Titanium dioxide nanoparticles induce emphysema-like lung injury in mice, FASEB J. 20 (2006) 2393-2395.

[64] M. Panduraqan, M. Veerappan, D.H. Kim, Cytotoxicity of zinc oxide nanoparticles on antioxidant enzyme activities and mRNA expression in the cocultured C2C12 and 3T3-L1Cells, Appl. Biochem. Biotechnol. 75 (2014) 1270-1280.

[65] A.K. Patlolla, D. Hackett, P.B. Tchounwou, Silver nanoparticle-induced oxidative stress-dependent toxicity in Sprague-Dawley rats, Mol. Cell. Biochem. 399 (2015) $257-268$.

[66] H.S. Jiang, X.N. Qiu, G.B. Li, W. Li, L.Y. Yin, Silver nanoparticles induced accumulation of reactive oxygen species and alteration of antioxidant systems in the aquatic plant Spirodela polyrhiza, Environ. Toxicol. Chem. 6 (2014) 1398-1405.

[67] S. Liu, L. Xu, T. Zhang, G. Ren, Z. Yang, Oxidative stress and apoptosis induced by nanosized titanium dioxide in PC12 cells, Toxicology 267 (2014) 172-177. 